\title{
EL EFECTO DE ENSEÑAR LAS DESTREZAS DEL PENSAMIENTO CRÍTICO EN UN CURSO INTRODUCTORIO DE ENFERMERÍA
}

Lydia Gordón de Isaacs*

En este estudio casi experimental se utilizó un modelo de enseñanza para el desarrollo del pensamiento crítico con un grupo de estudiantes de enfermería de primer año con el propósito de determinar si hubo adquisición de estas destrezas. Después de doce semanas de su implementación los resultados indicaron que el tratamiento fue estadísticamente significativo. Se concluye que el método de instrucción utilizado para promover las destrezas del pensamiento crítico parece efectivo en cursos introductorios de Enfermería.

UNITERMOS: enseñanza de enfermería, destreza de pensamiento crítico, avaluación de enseñanza

\section{INTRODUCCIÓN}

La atención primaria de salud, como estrategia para alcanzar el desarrollo de la salud, involucra entre otras cosas la reorientación del sistema de salud. Parte de esa reorientación lógicamente debe reflejarse también en los programas de formación de recursos humanos. Durante la década del 80 , se dio inicio a un importante movimiento de evaluación, análisis crítico y propuestas sobre la problemática de la educación en el área de salud. En 1985, en el Informe del Grupo de Consulta de Investigación sobre Personal de Salud se propuso la investigación del proceso educativo como una de las áreas prioritarias y se incluyó entre otros, el estudio y búsqueda de nuevas metodologías para la formación profesional (1).

\footnotetext{
* Maestría de la Universidad Nacional de Panamá. Facultad de Enfermería
} 
La función de la universidad en el fomento del concepto de Salud para todos en el 2000, puede expresarse en términos de las funciones tradicionales de la universidad: enseñanza, investigación y servicio. El reto que plantea a la enseñanza el fomento de este concepto está primero, en la necesidad de identificar las competencias que debe poseer y las actitudes que debe adoptar el personal de salud para operar de una manera eficaz en programas de atención de salud con un enfoque en atención primaria. Otra tarea consiste en lograr que los futuros profesionales de la salud posean la base científica necesaria y puedan aplicarla en la solución de los problemas de salud de la población a la que atienden (2).

Existe una gran preocupación porque ha pasado más de una década desde la reunión de Alma-Ata y los cambios han sido muy lentos en relación a la salud para todos. A menos de 10 años para llegar al 2000, y a pesar de que los países han hecho grandes esfuerzos por reformular las políticas de salud estamos muy distantes de la meta. La meta y la estrategia de atención primaria demandan cambios substanciales en todos los niveles del sistema de salud. Se requiere de actitudes, de niveles de competencia, de conocimiento y de destrezas diferentes a las que han caracterizado al personal de salud.

El proceso de cambio debe estar basado en el desarrollo de una capacidad de análisis y de crítica de los actuales servicios de salud y de las acciones y métodos del personal de salud.

En relación al personal de enfermería, se ha señalado que se necesita mayor liderazgo. Pensamos que se necesita un liderazgo crítico. Las enfermeras deben poseer la habilidad de abordar los diferentes enfoques de los problemas de salud que afectan a la población, deben hacer juicios críticos sobre los servicios de salud, deben buscar las mejores alternativas tomando en cuenta diferentes puntos de vista del equipo de salud y todos los facto res que influyen en la situación en un momento dado. Para formar enfermeras con características de líderes se hace necesario que se implementen cambios curriculares tendientes a promover el desarrollo de las destrezas del pensamiento crítico de las enfermeras.

La formación de la enfermera como líder debe comenzar des de el nivel de enseñanza de pre grado, donde se provea la oportunidad de desarrollar actitudes críticas, creativas y transformadoras. Los educadores de enfermería deben reflexionar sobre este aspecto en particular, el desarrollo del pensamiento crítico de nuestros estudiantes como herramienta para promover el liderazgo de enfermería.

Se ha dicho que la formación debe despertar interés en el estudiante por la aplicación del método científico y el método epidemiológico para el diagnóstico y análisis de los problemas de salud. Que debe estimularse el aprendizaje de técnicas de recolección de datos y utilización de los mismos, que se deben desarrollar conocimientos y habilidades esenciales para el 
desarrollo de un juicio clínico en los diversos contextos de la práctica de enfermería (3). En cierta medida eso quiere decir desarrollar el pensamiento crítico.

Cornbleth (1985), identificó la esencia del concepto de pensamiento crítico señalando que es un escepticismo informado, una búsqueda activa de la verdad, en lugar de una aceptación pasiva de la tradición, de la autoridad o de lo que es común. Dice que es un proceso dinámico de preguntar, de razonar, de cuestionar las conclusiones, definiciones, creencias y acciones (4).

Villalobos (1988), señala que pareciera que la mayoría de los programas básicos de enfermería permanecen dentro de esquemas tradicionales de formación, a pesar de los muchos esfuerzos que se han llevado a cabo con el fin de modificar los enfoques meramente intrahospitalarios hacia aquellos comunitarios y de atención primaria. A su modo de ver, se debe dar mayor prioridad a los procesos cognoscitivos y las habilidades intelectuales de los estudiantes de enfermería (5).

Pensamos que si se incluye el desarrollo de las destrezas del pensamiento crítico como parte integral de la formación de las enfermeras, podría repercutir directamente en el fomento de mayor liderazgo en enfermería.

\section{EL ESTUDIO}

En este estudio las destrezas del pensamiento crítico fueron enseñadas en un curso introductorio de enfermería. El estudio estaba basado en la concepción de pensamiento crítico de Robert Ennis. Según este autor, el pensamiento crítico es definido como "el pensamiento reflexivo y razonable que se enfoca en decidir que creer o que hacer" (Ennis, 1985, p. 45). De acuerdo a Ennis hay muchas formas de subcategorizar el pensamiento crítico. Su enfoque distingue tres formas de inferencias para las creencias (inducción, deducción y evaluación), y cuatro tipos de bases para tales inferencias: los resultados de otras inferencias, observaciones, declaraciones hechas por otros y supuestos (6).

El problema planteado fue como enseñar a los estudiantes con el fin de mejorar sus destrezas de pensamiento crítico. ¿Sería posible enseñar las destrezas del pensamiento crítico como parte de un curso introductorio de Enfermería y lograr la adquisición de estas destrezas en los estudiantes?

\section{PROPÓSITOS DEL ESTUDIO}

1. Proveer una traducción al Español, confiable y válida, del instrumento Prueba Cornell para Pensamiento Crítico. Nivel Z. 
2. Determinar el efecto en la adquisición de destrezas de pensamiento crítico al enseñar a estudiantes de enfermería un curso introductorio de enfermería con enfoque en el desarrollo de destrezas de pensamiento crítico.

\section{PREGUNTAS DE LA INVESTIGACIÓN E HIPÓTESIS}

1. ¿Cuales son las propiedades psicométricas de la versión en español de la Prueba Cornell para Pensamiento Crítico? ¿Nivel Z?

2. ¿Podrán los estudiantes de enfermería a los cuales se les enseña Enf. 103 utilizando el modelo A., para el desarrollo del pensamiento crítico obtener un puntaje mayor en la prueba de pensamiento crítico que los estudiantes que son enseñados Enf. 103 utilizando el Modelo B. o método convencional?

\section{HIPÓTESIS}

Los estudiantes a los cuales se les enseña un curso introductorio de Enfermería siguiendo el Modelo Educativo (A) para el pensamiento crítico obtendrán un puntaje más alto en la prueba Cornell para Pensamiento Crítico Nivel $Z$ que los estudiantes de Enfermería a los cuales se les enseña el mismo curso con el modelo (B) o tradicional.

\section{DISEÑO}

El diseño de este estudio fue casi-experimental (Pre-test - Post-test), ya que los grupos se formaron voluntariamente y fueron asignados al azar para tratamiento y comparación.

\section{PROCEDIMIENTOS}

Para poder medir los efectos de enseñar para el desarrollo del pensamiento crítico fue necesario traducir al español la Prueba Cornell para pensamiento Crítico Nivel Z, luego de obtener el permiso del autor. Debido a que cada idioma tiene sus peculiaridades y modos 
de expresión que no pueden ser expresados con una simple traducción fue necesario hacer unas adaptaciones al instrumento. El instrumento traducido y adaptado fue examinado por equivalencia por dos profesores de la escuela de lenguas de la Universidad de Miami y por una consultora de educación con experiencia en programas de pensamiento crítico.

Al traducir y adaptar un instrumento a un nuevo idioma y cultura las propiedades psicométricas de la nueva versión deben ser estudiadas (7). Para examinar las propiedades psicométricas de la versión en Español de la prueba Cornell. Nivel Z fue necesario aplicarlo a 120 estudiantes de la Facultad de Enfermería Universidad de Panamá. Los datos obtenidos fueron analizados para determinación de variabilidad (media, desviación estándar, determinación de índice de confiabilidad).

\section{TRATAMIENTO}

Un modelo para enseñar las destrezas del pensamiento crítico fue desarrollado basada en el concepción de pensamiento crítico de Ennis (8). Este modelo incluyó ocho destrezas de pensamiento crítico que fueron integradas al contenido de un curso introductorio de Enfermería y enseñado por doce semanas.

Las destrezas enseñadas fueron:

- Analizar argumentos. Se entiende por argumento una forma de pensar en que se dan ciertas razones para apoyar una conclusión. Se utilizaron criterios para analizar los argumentos.

- Juzgar la credibilidad de una fuente de información. Para esta destreza se analiza la fuente de información y se decide si la fuente es creíble, se usan criterios específicos.

- Juzgar reportes de observaciones. Donde se determina la objetividad y validez de reportes escritos que se presentan, este juicio se hace en base a criterios.

- Juzgar deducciones. Se Juzgan argumentos deductivos, es decir, argumentos en que se razona de premisas que se sabe o se asume que son ciertas a una conclusión que sigue lógicamente de las premisas.

- Juzgar inducciones. Se juzgan argumentos inductivos, es decir, generalizaciones inductivas, hipótesis explicativas, y sistemas teóricos. Se establece una diferencia entre los diferentes tipos de inducciones y se usan criterios específicos. 
- Definir términos y juzgar definiciones. Donde se revisa la habilidad de definir con claridad y precisión; y se juzgan definiciones de acuerdo a ciertos criterios.

- Identificar supuestos. Un supuesto es definido como algo que se toma como un hecho. Se sostiene que para entender el punto de vista de un autor hay que identificar los supuestos implícitos en los argumentos presentados por el autor. Se identifican supuestos en base a criterios.

- Decidir sobre una acción. Se buscan diferentes alternativas para un problema y se selecciona la mejor. Se usan criterios para dicha selección.

Para enseñar cada destreza se siguieron tres pasos: introducción de la destreza, explicación de la destreza y demostración de la misma. Estos pasos fueron seguidos por un período de práctica con guía del profesor. Los estudiantes trabajaron en pequeños grupos y fueron asistidos cuando requerían ayuda. Al final de cada sesión, un estudiante designado por el grupo de trabajo presentaba a todo el grupo 10 que habían hecho.

Debido a que el pensamiento no ocurre en el vacío, era muy importante familiarizar a los estudiantes con el contenido de la materia antes de enseñarles las destrezas de pensamiento crítico. Para poder lograr esto, un período de 45 minutos semanales fueron usados para clases magistrales sobre el contenido del curso seguidos de 15 minutos para preguntas y clarificación de la clase. Se asignaba la lectura del material presentado (de apuntes del curso y literatura seleccionada). Los últimos 30 minutos del período eran usados para introducir, explicar y demostrar la

destreza utilizando material del contenido del curso.

La clase se reunía dos veces a la semana por una hora y media. En la segunda reunión, los primeros 50 minutos eran usados para una actividad en grupo para practicar la destreza que fue introducida explicada y demostrada en la clase anterior. La actividad usaba material del contenido de la clase anterior que también había sido asignado para lectura. Los últimos 40 minutos se usaban para que el estudiante designado del grupo presentara los resultados de su grupo de trabajo a la clase.

El grupo de comparación fue enseñado dando clases sobre el contenido del curso en los primeros 45 minutos de la primera hora semanal, los 15 minutos siguientes se hacían preguntas y se clarificaba el material presentado. El material se asignaba para lectura para la próxima clase. Los últimos 30 minutos eran usados para iniciar la actividad de grupo que realizarían en la próxima clase. Los primeros 50 minutos de la siguiente clase eran usados para continuar la actividad de grupo organizada en la clase anterior, y los últimos 40 minutos se usaron para las presentaciones de los grupos a la clase. Solamente en el grupo experimental se usó el modelo educativo que a continuación presentamos. 


\section{EXPLICACIÓN DEL CONTENIDO DE LA MATERIA}

DISCUSIÓN Y PREGUNTAS DEL CONTENIDO

INTRODUCCIÓN DE LA DESTREZA

EXPLICACIÓN DE LA DESTREZA

DEMOSTRACIÓN DE LA DESTREZA (UTILIZANDO CONTENIDO

DEL CURSO)

FORMACIÓN DE PEQUENOS GRUPOS

PRÁCTICA DE LA DESTREZA (UTILIZANDO CONTENIDO DEL CURSO)

PRESENTACIÓN DE RESULTADOS A LA CLASE (POR UN ESTUDIANTE DE CADA PEQUEÑO GRUPO)

ESQUEMA DEL MODELO EDUCATIVO PARA LA INTEGRACIÓN DEL PENSAMIENTO CRÍTICO EN LAS DISCIPLINAS. FUENTE: DRA. LYDIA DE ISAACS

\section{MUESTRA}

La muestra inicial estuvo formada por 70 estudiantes registrados para Enf 103, un curso del segundo semestre del primer año. El curso está dividido en 4 horas académicas. Este curso es un requisito para el programa de 4 años de licenciatura en ciencias de Enfermería de la Facultad de Enfermería de la Universidad de Panamá. Todos los estudiantes registrados en este programa tienen un título secundario de Bachiller en Ciencias, y para matricularse deberán haber pasado todos los cursos del primer semestre del primer año. El estudiante promedio que inicia este programa está entre 18 a 25 años y son en su mayoría del sexo femenino.

Al inicio del semestre dos grupos de 35 estudiantes registrados fueron tornados y asignados al azar uno a tratamiento y el otro para comparación. Durante la primera semana de clases a ambos grupos se les aplicó la prueba de Cornell para Pensamiento Crítico Nivel $\mathrm{Z}$ traducida previamente al Español. 
El grupo de tratamiento fue enseñado usando el Modelo A para el desarrollo del pensamiento crítico y el grupo de comparación fue enseñado con el modelo B o método convencional.

La prueba de Cornell para el Pensamiento Crítico fue aplicada nuevamente a ambos grupos al finalizar el semestre.

\section{ANÁLISIS DE DATOS}

Los datos fueron analizados por el método de análisis de covarianza, usando el pretest como la covarianza. Se usó un nivel de confianza de 0.05. Las medias ajustadas fueron usadas para evaluar la efectividad del tratamiento. Las variables independientes fueron los métodos de instrucción y la variable dependiente la de adquisición de destrezas de pensamiento crítico según la prueba de Cornell de Pensamiento Crítico Nivel Z (Versión en Español).

\section{SIGNIFICANCIA DEL ESTUDIO}

Este es el primer estudio en la Universidad de Panamá que examina los erectos de enseñar las destrezas del pensamiento crítico. Es la primera respuesta a la necesidad de estudios empíricos sobre la enseñanza del pensamiento crítico en este país. Sin estos datos es muy difícil saber que tipo de instrucción en pensamiento critico es valioso para los estudiantes y si esta instrucción esta siendo efectiva.

La tabla 1 muestra la media y desviación estándar para los puntajes en la Prueba para el pre-test y el post-test de ambos grupos. Se observa una gran ganancia en puntaje entre el pre-test y el post-test del grupo experimental.

\section{TABLA 1 - DESVIACIÓN ESTÁNDAR Y MEDIAS PARA LOS GRUPOS A Y B}

\begin{tabular}{ccrr}
\hline Grupo & Número & Pre-test & \multicolumn{1}{c}{ Post-test } \\
\hline A & 30 & M 24.63 & M.31.97 \\
& & *Med. ajust 31.86 \\
B & 30 & DS 3.67 2.91 \\
& & M 23.40 & M.23.17 \\
& & DS 3.89 & DS2.95 \\
\hline
\end{tabular}

\footnotetext{
* Med. ajust medidas de post-test ajustadas
} 
Las medias ajustadas fueron usadas para el análisis de covarianza. (ANCOVA usa las medias ajustadas para ajustar los grupos por diferencias iniciales).

TABLA 2 - ANCOVA PARA PUNTAJES EN LA PRUEBA CORNELL PARA PENSAMIENTO CRÍTICO NIVEL Z CON EL PRE-TEST

\begin{tabular}{lccrc}
\hline Fuente de variación & SC & gl & MC & \multicolumn{1}{c}{ F } \\
\hline Covarianza (Pre t.) & 108.654 & 1 & 108.654 & $13.094^{*}$ \\
Efecto principal & 1077.110 & 1 & 1077.110 & 129.800 \\
Residuo & 472.733 & 57 & 8.298 & \\
Total & 1658.733 & 59 & & \\
\hline
\end{tabular}

$* p<.01$

El análisis de covarianza con el pre-test como covarianza fue usado para probar la hipótesis nula de no diferencia significativa entre el grupo experimental y el grupo de comparación. El análisis (tabla 2) reveló una diferencia significativa para el tratamiento ( $p<$ .01). La hipótesis nula no diferencia fue rechazada. La hipótesis de investigación que decía que el grupo experimental obtendría mayor puntaje fue aceptada.

La linealidad de la covarianza con la variable dependiente, un supuesto necesario para realizar análisis de covarianza como un método estadístico aceptable, fue cumplido. La covarianza estaba significativamente relacionada a la variable dependiente $(F(1,57)=$ $13.0947 \mathrm{p}<.01)$.

El supuesto de homogeneidad de la regresión o sea ninguna interacción entre la covarianza y el tratamiento también fue cumplido $(F(1,56)=.18 p=.67)$.

\section{LIMITACIONES}

Una preocupación desde el inicio del estudio era como los retiros del curso afectarían la muestra final, para ello se planeó una muestra final de 30 estudiantes a pesar de que se inició con una muestra de 35 estudiantes por grupo. Otra consideración era el hecho de que podría haber variación entre la personalidad, motivación, e interés de los profesores que enseñaban el curso. Para controlar esto, la investigadora enseño tanto al 
grupo experimental, como al grupo de comparación, reconociendo por otro lado, que el estudio puede ser criticado por sesgo del investigador al enseñar ambos grupos.

Finalmente pero no menos importante, otra preocupación fue el hecho de que por razones fuera del control de la investigadora, el tratamiento fue interrumpido por ocho semanas, luego continuado por siete semanas académicas después de las cuales se administró el post-test. No sabemos con seguridad como este hecho ha afectado los resultados obtenidos.

\section{DISCUSIÓN}

El pensamiento crítico envuelve operaciones mentales, disposiciones y conocimientos (9). Las destrezas envueltas pueden ser enseñadas. Por ejemplo, Dewey (1910) creyó que los estudiantes podían desarrollar su habilidad para pensar a través de la educación, el defendió la educación basada en el método científico integrando experiencia y reflexión con el aprendizaje del contenido (10). Ennis ( 1985) sostiene que pensar críticamente envuelve destrezas, conocimientos y disposiciones y que la habilidad de los estudiantes de pensar críticamente puede ser mejorada enseñando a hacerlo, el identificó las destrezas del pensamiento crítico (11) .Estas destrezas pueden ser integradas en un curso teórico. Los resultados de este estudio sugieren que estas ocho destrezas de pensamiento crítico pueden ser enseñadas como parte de un curso introductorio de enfermería.

Muchos investigadores sostienen que el pensamiento crítico debe ser enseñado en cursos genéricos especialmente diseñados para enseñar a pensar críticamente. En relación a este punto, Kurfiss (1988), sostiene que la limitación fundamental de los cursos genéricos de pensamiento crítico es que las preguntas que se hacen determinan el valor de una investigación y que sin conocimiento de la materia a investigar es difícil hacer preguntas inteligentes (12). Los resultados de este estudio proveen datos empíricos sobre la efectividad de enseñar las destrezas de pensamiento crítico integradas en el contenido de enfermería.

A pesar de que Beyer (1988) señala la necesidad de introducir una pequeña cantidad de destrezas en un período de tiempo (13), en este estudio se introdujeron 8 destrezas en 12 semanas. El modelo educativo utilizado resultó ser efectivo para esta muestra de estudiantes de enfermería de primer año.

Los resultados de este estudio son consistentes con evidencia de otros estudios que demuestran que la habilidad de pensar críticamente puede ser substancialmente aumentada 
por medio de instrucción y integración de la enseñanza del pensamiento crítico en el curriculum (14). Muchos estudios indican que la mayoria de las personas no desarrollan la proficiencia de realizar operaciones de pensamiento formal tales como las de pensamiento crítico en una forma automática $(13,14,15,16,17)$. Los educadores debemos enseñar para que los estudiantes desarrollen el pensamiento crítico porque no ocurre automaticamente. David Perkins nos indica que "el arte de pensar es como correr las 100 yardas, es una habilidad técnica llena de artificios" (18).

\section{CONCLUSIONES}

Este estudio presenta un modelo para enseñar las destrezas del pensamiento crítico integrado en el contenido de enfermería. Debe ser considerado como una respuesta a la necesidad de estudios empíricos sobre modelos de enseñanza para el desarrollo de las destrezas de pensamiento crítico. Dentro de las limitaciones de este estudio las siguientes conclusiones parecen válidas:

1. El instrumento Cornell para pensamiento crítico. Nivel Z traducido al español es un instrumento válido y confiable. Este instrumento constituye un aporte importante para la investigación y educación del pensamiento crítico en Panamá y en otros países latinoamericanos.

2. El método de instrucción usado para promover las destrezas de pensamiento crítico es efectivo para enseñar estas destrezas integradas a cursos introductorios de Enfermería.

\section{RECOMENDACIONES PARA FUTURAS INVESTIGACIONES}

El modelo ofrece oportunidad para futuros estudios al aplicarlo en otros cursos de Enfermería a diferentes niveles. Además, la réplica del estudio con otras muestras de estudiantes de primer año con resultados similares añadiría robustez a este estudio. Existe la necesidad de investigaciones que demuestren la efectividad de diferentes métodos de enseñar a pensar en cursos introductorios de diferentes disciplinas de la salud. También sugerimos la necesidad de investigaciones que vean la relación entre aprendizaje del contenido del curso y aprendizaje de destrezas de pensamiento crítico. Se recomienda también el estudio de las condiciones instruccionales e institucionales que favorezcan la 
investigación crítica. Se necesitan además, investigaciones que revelen como se origina la disposición para pensar críticamente. Específicamente sugerimos la necesidad de estudios que examinen el efecto del maestro como modelo de una persona que piensa críticamente. Por otro lado, las investigaciones sobre las creencias de los estudiantes y sus estrategias de aprendizaje, pueden ser útiles para entender las complejidades de la enseñanza para el desarrollo de las destrezas del pensamiento crítico.

\section{THE RESULT OF TEACHING CRITICAL THOUGHT SKILLS IN A INTRODUCTORY NURSING COURSE}

This almost-experimental study utilized an education model to develop critical thought in a group of first year nursing students having as an objective to determine skills acquisition. Twelve weeks after its implementation the results showed the treatment statistical significance. Therefore, the instruction method utilized to promote critical thought skills produced the intended result in introductory nursing courses.

UNITERMS: nursing education, critical thought skills, education evaluation

\section{REFERÊNCIAS BIBLIOGRÁFICAS}

01. ORGANIZACIÓN PANAMERICANA DE LA SALUD/PROGRAMA DE DESARROLLO DE RECURSOS HUMANOS. Investigación de personal de salud. Washington, DC, $\mathrm{n}$. 66, p. 60-61, 1985.

02. ORGANIZACIÓN PANAMERICANA DE LA SALUD. Función de las universidades en las estrategias de salud para todos. Educación Médica y Salud. Washington, DC, v. 19, n. 1, 102 p., 1985.

03. ORGANIZACIÓN PANAMERICANA DE LA SALUD. Leadership for health for all. The Challenge to nursing: a strategy for action. Publicación 86.1, 1987.

04. CORNBLETH, C. Critical thinking and cognitive process. In: W. Stanley, ed., Review of

Research in Social Studies Education 1976-1983. Washington, DC, National Council for the Social Sciences, 1985. 
05. VILLALOBOS, M. El desarrollo del liderazgo y la educación en Enfermería. Educación Médica y Salud. v. 22, n. 2, p. 191-199, 1988.

06. ENNIS, R. A logical basis for measuring critical thinking skills. Educational Leadership. V. 43, n. 2, p. 44-48, 1985.

07. AMERICAN EDUCATIONAL RESEARCH ASSOCIATION / AMERICAN PSYCHOLOGICAL ASSOCIATION / NATIONAL COUNCIL ON MEASUREMENT IN EDUCATION. Standards for Educational and Psychological Testing. Washington, DC, American Psychological Association. 1985.

08. ENNIS, R. ibid.

09. BEYER, B. Developing a thinking skills program. Boston, Allyn Bacon, Inc. 1988.

10. DEWEY, J. How we think. Boston, D.C. Health \& Co. 1910.

11. ENNIS, R. ibid.

12. KURFISS, J. Critical thinking, theory, research, practice and possibilities. Washington. ASHE-ERIC. 1988.

13. BEYER, B. ibid.

14. GLASER, R. Education and the role of knowledge. American Psychologist. V. 13, n. 9, p. 5-10. 1984.

15. REUVEN, F. Instrumental Enrichment: An Intervention Program for Cognitive Modifiability. Baltimore: University Press. 1980.

16. WHIMBEY, A., LOCKHEAD, J. Problem Solving and Comprehension, 3. ed., Philadelphia: Franklin Institute Press. 1987.

17. PEA, R., KURTLAND, M. On the cognitive effects of learning computer program. New Ideas in Psychology. V. 2, n. 2, p. 37-168. 1984.

18. PERKINS, D. Reasoning as it is and it should be: An empirical perspective. Paper presented at the National Conference of the American Educational Research Association. San Francisco. 1986. 